\title{
FOREWORD
}

\section{Special Section on Software Defined Radio Technology and Its Applications}

Based on the successful fundamental research and development (R\&D) on software defined radio (SDR) until 2005, the word "SDR" has become popular and several actual prototypes based on SDR for several usage models such as cellular communication systems, wireless LAN, PAN, ITS and so on have already been developed in all over the world. This was the first stage of R\&D on SDR. The IEICE Technical Committee of Software Radio (TCSR) has contributed to promoting the first-stage R\&D since 1998. From the end of 2005 , the R\&D has stood on the second stage, because a new research topic "cognitive radio" that has very close relationship with SDR is activated and multi-mode RF devices are ready for use as consumer products in order to support the new radio communication system. To make the second stage a success, more frequent information-exchanges between key technologies such as radio frequency part, digital signal processing part, and cross-layer communication part must be needed. To promote the information-exchanges, the publication of this special section was planned with a view to promoting further progress of R\&D in this field.

In reply to call for papers, 21 submissions are received. After impartial review, 3 invited papers related to reconfigurable device, RF device, and cognitive radio, 9 papers and 2 letters are accepted for publication in this section. The papers cover topical subjects such as implementation architecture, system implementation on FPGA, ADC, filter design for SDR, and channel recognition. I am confident that the papers will be instructive to the people who are engaged in R\&D on SDR.

Finally, I would like to thank to express my sincere application to all authors for their contributions and to many reviewers and this section's editorial committee members, Ryuji Kohno (Yokohama National University), Hideharu Amano and Yukitoshi Sanada (Keio University), Tetsushi Ikegami (Meiji University), Yoshio Kunisawa (KDDI Lab.), Noriharu Suematsu (Mitsubishi Electric), Kazuhiro Uehara (NTT), Satoshi Denno (Kyoto University), Hidekazu Murata (Tokyo Institute of Technology), and Hitoshi Yoshino (NTT DoCoMo) for their voluntary activities. Also I would like to express my cordial appreciation to Kei Sakaguchi (Tokyo Institute of Technology) and Takeo Fujii (The University of Electro-Communications) for their excellent management as secretaries of this section.

Hiroshi Harada, Guest Editor-in-Chief

\begin{abstract}
Hiroshi Harada (Member) received Ph.D. degree from Osaka University, Osaka, Japan in 1995. In 1995, he joined the Communications Research Laboratory (CRL), Ministry of Posts and Telecommunications (MPT), Tokyo (now National Institute of Information and Communications Technology (NICT)). He is currently the research manager of ubiquitous mobile communications group of New Generation Wireless Communications Research Center, NICT, and is also the chair of Technical Committee of Software Radio, IEICE Communication Society from May 2005. His current research interests include digital-signal-processing based mobile telecommunication systems, e.g. software radio, cognitive radio and broadband wireless access (BWA) systems. Dr. Harada received the Excellent Paper Award of the Third International Symposium on Wireless Personal Multimedia Communications (WPMC) in 2000 and 2006, YRP Award in 2002, the Young Scientist Award from the Minister of Education, Culture Sports Science and Technology (MEXT), Japan, and the achievement award of IEICE
\end{abstract}

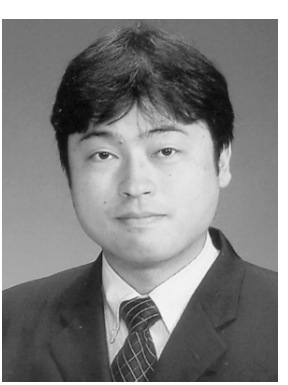
in 2006. He is a co-author of "Simulation and Software Radio for Mobile Communication" (Artech House, 2002). 